\title{
Effects of the PCSK9 antibody alirocumab on coronary atherosclerosis in patients with acute myocardial infarction: a serial, multivessel, intravascular ultrasound, near-infrared spectroscopy and optical coherence tomography imaging study-Rationale and design of the PACMAN-AMI trial
}

Christian Zanchin, MD, PhD ${ }^{\mathrm{a}, 1}$, Konstantinos C. Koskinas, MD ${ }^{\mathrm{a}, 1}$, Yasushi Ueki, MD ${ }^{\mathrm{a}}$, Sylvain Losdat, $\mathbf{P h D}^{\mathrm{b}}$, Jonas D. Häner, MD ${ }^{\mathrm{a}}$, Sarah Bär, MD ${ }^{\mathrm{a}}$, Tatsuhiko Otsuka, MD ${ }^{\mathrm{a}}$, Andrea Inderkum, MSc ${ }^{\mathrm{a}}$, Maria Radu Juul Jensen, MD, $\mathbf{P h D}^{\mathrm{c}}$, Jacob Lonborg, $\mathbf{M D}^{\mathrm{c}}$, Gregor Fahrni, $\mathbf{M D}^{\mathrm{d}}$, Anna S. Ondracek ${ }^{\mathrm{e}}$, Joost Daemen, MD ${ }^{\mathrm{f}}$, Robert-Jan van Geuns, $\mathrm{MD}^{\mathrm{g}}$, Juan F. Iglesias, $\mathrm{MD}^{\mathrm{h}}$, Christian M. Matter, MD $^{\mathrm{i}}$, David Spirk, MD ${ }^{\mathrm{j}}$, Peter Juni, MD ${ }^{\mathrm{k}}$, Francois Mach, $\mathbf{M D}^{\mathrm{h}}$, Dik Heg, $\mathbf{P h D}^{\mathrm{b}}$, Thomas Engstrom ${ }^{\mathrm{c}}$, Irene Lang $^{\mathrm{e}}$,

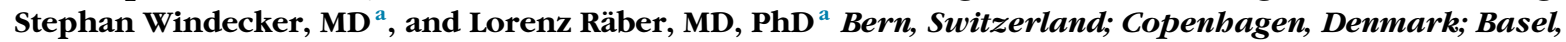
Switzerland; Vienna, Austria; Rotterdam, The Netherlands; Nijmegen, The Netherlands; Geneva, Switzerland; Zurich, Switzerland; Ontario, Canada

Background The risk for cardiovascular adverse events after acute myocardial infarction (AMI) remains high despite potent medical treatment including low-density lipoprotein cholesterol (LDL-C) lowering with statins. Proprotein convertase subtilisin/kexin type 9 (PCSK9) antibodies substantially reduce LDL-C when added to statin. Alirocumab, a monoclonal antibody to PCSK9, reduces major adverse cardiovascular events after AMI. The effects of alirocumab on coronary atherosclerosis including plaque burden, plaque composition and fibrous cap thickness in patients presenting with AMl remains unknown.

Aims To determine the effect of LDL-C lowering with alirocumab on top of high-intensity statin therapy on intravascular ultrasound (IVUS)-derived percent atheroma volume (PAV), near-infrared spectroscopy (NIRS)-derived maximum lipid core burden index within $4 \mathrm{~mm}\left(\mathrm{maxLCBI}_{4 \mathrm{~mm}}\right)$ and optical coherence tomography (OCT)-derived fibrous cap thickness (FCT) in patients with AMI.

Methods In this multicenter, double-blind, placebo-controlled trial, 300 patients with AMI (ST-elevation or non-STelevation myocardial infarction) were randomly assigned to receive either biweekly subcutaneous alirocumab (150 mg) or placebo beginning $<24$ hours after the acute event as add-on therapy to rosuvastatin $20 \mathrm{mg}$. Patients undergo serial IVUS, NIRS and OCT in the two non-infarct related arteries at baseline (at the time of treatment of the culprit lesion) and at 52 weeks. The primary endpoint, change in IVUS-derived PAV, and the powered secondary endpoints, change in NIRS-derived $\operatorname{maxLCBI}_{4 \mathrm{~mm}}$, and OCT-derived minimal FCT, will be assessed 52 weeks post randomization.

Summary The PACMAN-AMI trial will determine the effect of alirocumab on top of high-intensity statin therapy on high-risk coronary plaque characteristics as assessed by serial, multimodality intracoronary imaging in patients presenting with AMI.

From the a Department of Cardiology, Inselspital, Bern University Hospital, University of Bern, Bern, Switzerland, b/nstitute of Social and Preventive Medicine and Clinical Trials Unit, Bern University Hospital, Bern, Switzerland, ' Department of Cardiology, Rigshospitalet, Copenhagen University Hospital, Copenhagen, Denmark, ${ }^{d}$ Department of Cardiology, University Hospital Basel, Basel, Switzerland, e Department of Cardiology, Medical University of Vienna, Vienna, Austria, ' Department of Cardiology, Erasmus Medical Center, Rotterdam, The Netherlands, 9 Department of Cardiology, Radboud UMC, Nijmegen, The Netherlands, hDepartment of Cardiology, Geneva University Hospital, Geneva, Switzerland, 'Department of Cardiology, University Heart Center Zurich, University Hospital Zurich, Zurich, Switzerland, iDepartment of Pharmacology, Bern University Hospital, Bern, Switzerland and Sanofi, Switzerland, kDepartment of Medicine and
Institute of Health Policy, Management and Evaluation, University of Toronto, Toronto, Ontario, Canada

${ }^{1}$ Equally contributing first authors.

Submitted November 16, 2020; accepted April 26, 2021

Reprint requests: Lorenz Räber, MD, PhD, Department of Cardiology, Bern University Hospital, University of Bern, 3010 Bern, Switzerland

E-mail address: lorenz.raeber@insel.ch. 0002-8703

(C) 2021 Published by Elsevier Inc.

https://doi.org/10.1016/i.ahi.2021.04.006 


\section{Clinical trial registration NCT03067844 (Am Heart J 2021;238:33-44.)}

\section{Background}

Increased residual risk in patients after acute myocardial infarction

Lowering plasma levels of low-density lipoprotein cholesterol (LDL-C) significantly reduces cardiovascular mortality and morbidity. ${ }^{1,2}$ Despite advances in coronary revascularization and medical treatment, the risk for recurrent cardiovascular events remains high, especially in patients who suffered a recent acute myocardial infarction (AMI). While statins represent a first-line therapy for secondary prevention ${ }^{3}$, more than $50 \%$ of patients treated with statins do not achieve their target LDL-C levels or cannot tolerate effective statin doses. ${ }^{4}$ Accordingly, substantial LDL-associated residual risk remains. Monoclonal antibodies inhibiting the proprotein convertase subtilisin/kexin type 9 (PCSK9) enzyme have emerged as a valuable add-on therapy in patients who require additional LDL-C lowering despite statin therapy. PCSK9 inhibitors lower LDL-C levels by approximately $60 \%$ and reduce major adverse cardiovascular events in stable patients with atherosclerotic cardiovascular disease (ASCVD) ${ }^{5}$ and following acute coronary syndromes (ACS). ${ }^{6}$ The EVOPACS study demonstrated safe and profound LDL-C lowering with evolocumab in the acute setting of ACS. ${ }^{7}$

Coronary atherosclerosis is characterized by substantial heterogeneity ranging from minor subintimal lipid depositions to large fibrous or fibrocalcific plaques to highly inflamed, rupture-prone thin-capped fibroatheromas. In recent years, intracoronary imaging modalities have enabled a detailed evaluation of human coronary atherosclerosis beyond the "lumenogram" provided by coronary angiography. ${ }^{8}$ Intravascular ultrasound (IVUS) allows for accurate quantification of atherosclerotic plaque burden. ${ }^{9}$ Due to its high resolution, optical coherence tomography (OCT) can measure fibrous cap thickness (FCT), which is a determinant of plaque vulnerability. ${ }^{10}$ Near-infrared spectroscopy NIRS has been validated in coronary autopsy specimens ${ }^{11}$ and subsequently in vivo ${ }^{12}$ for the detection and quantification of lipid content within the coronary arteries. Taken together, these modalities provide complementary insights into the burden, compositions, and thereby the risk profile of coronary plaques in vivo. Of prognostic relevance, IVUS-, OCT-, and NIRS-defined measurements have been shown to predict future cardiovascular events in patients with established coronary artery disease (CAD). ${ }^{13-15}$

Statins halt atherosclerotic plaque progression and achieve regression when the highest doses are administered. ${ }^{16,17}$ Moreover, statins favorably affect plaque mor- phology and composition by reducing lipid content, attenuating plaque inflammation and increasing FCT of fibroatheromas. ${ }^{18-22}$ The GLAGOV randomized controlled trial assessed the effect of evolocumab vs placebo on top of statin in 896 patients with a coronary stenosis between $20 \%$ to $50 \% .{ }^{23}$ Percent atheroma volume (PAV) reduction after 18 months was greater in evolocumabtreated patients. In a substudy using virtual histology IVUS, there was no difference in the reduction of calcium, fibrous, fibrofatty, or necrotic tissue between groups. ${ }^{24}$ In a Japanese study ${ }^{25}, 206$ patients with LDL$\mathrm{C} \geq 2.59 \mathrm{mmol} / \mathrm{L}(\geq 100 \mathrm{mg} / \mathrm{dL})$ under stable statin therapy and a recent (2-4 wk) ACS were randomized to alirocumab or standard of care (atorvastatin $>10 \mathrm{mg} / \mathrm{d}$ or rosuvastatin $>5 \mathrm{mg} / \mathrm{d}$ ) for 36 weeks in an unblinded fashion. There was no difference in the change in total atheroma volume at 6 months. Possible explanations for the observed lack of effect might include the limited sample size, short treatment period, imaging of the infarct vessel, statin and non-statin up titration in the control group only, and introduction of ezetimibe in nearly half of patients in the control arm.

Collectively, the efficacy of statins in reducing coronary atheroma burden and favorably altering plaque composition is well established. However, while evolocumab has been shown to reduce plaque burden, there is a gap of evidence regarding the impact of PCSK9 inhibition on changes of presumed vulnerable plaque features, including morphology and composition by means of serial multimodality imaging - particularly among patients at highest risk, notably those with AMI. The PACMAN-AMI trial is a multi-modality intracoronary imaging study aiming to show the superiority of alirocumab vs. placebo, on top of high-intensity statin, on serial changes of IVUS-derived PAV, OCT-derived minimal FCT, and NIRSderived maximum lipid core burden index within $4 \mathrm{~mm}$ $\left(\operatorname{maxLCBI}_{4} \mathrm{~mm}\right)$ in the non-infarct-related arteries of patients with AMI undergoing PCI. Enrolment of 294 patients is scheduled. Therefore, PACMAN-MI will be the first intracoronary imaging trial to comprehensively investigate the entire spectrum of plaque modification by PCSK9 inhibition including volume, composition and microstructure.

\section{Methods}

Study design, study population, drug administration, and visit schedule

The effects of the PSCK9 antibody AliroCuMab on coronary Atherosclerosis in patieNts with Acute Myocardial Infarction trial (PACMAN-AMI, clinicaltrial.gov 
Table I. Inclusion and exclusion criteria

Inclusion criteria - Male or female, age $\geq 18$ y at screening

- Acute myocardial infarction: acute ST-segment elevation myocardial infarction (STEMI) with pain onset within $\leq 24 \mathrm{~h}$, or

non-ST segment elevation myocardial infarction (NSTEMI), with at least one coronary segment (culprit lesion) requiring PCI

- LDL-C $\geq 70 \mathrm{mg} / \mathrm{dL}(\geq 1.8 \mathrm{mmol} / \mathrm{L})$ assessed prior to, or during $\mathrm{PCl}$ in patients who have been receiving any stable statin regimen within $\geq 4$ wk prior to enrolment; $\underline{O R} L D L-C \geq 125 \mathrm{mg} / \mathrm{dL}(\geq 3.2 \mathrm{mmol} / \mathrm{L})$ in patients who are statin-naïve or have not been on stable statin regimen for $\geq 4 \mathrm{wk}$ prior to enrollment

- At least two major native coronary arteries ("target vessels") each meeting the following criteria for intracoronary imaging immediately following the qualifying $\mathrm{PCl}$ procedure:

- Angiographic evidence of $<50 \%$ reduction in lumen diameter by angiographic visual estimation

- Target vessel deemed to be accessible to imaging catheters and suitable for intracoronary imaging in the proximal $(50 \mathrm{~mm})$ segment ("target segment")

- Target vessel may not be a bypass (saphenous vein or arterial) graft or a bypassed native vessel

- Target vessel must not have undergone previous $\mathrm{PCl}$ within the target segment

- Target vessel is not candidate for intervention at the time of qualifying PCl or over the following 6 mo in the judgment of the Investigator

- Hemodynamic stability allowing the repetitive administration of nitroglycerine

- Ability to understand the requirements of the study and to provide informed consent

- Willingness of patient to undergo follow-up intracoronary imaging

Exclusion criteria Left-main disease, defined as $\geq 50 \%$ reduction in lumen diameter of the left main coronary artery by angiographic visual estimation

- Three-vessel disease, defined as $\geq 70 \%$ reduction in lumen diameter of three major epicardial coronary arteries by angiographic visual estimation or in major branches of one or more of these arteries, irrespective of the localization (proximal $50 \mathrm{~mm}$ or more distal localization) of the obstructive lesions

- History of coronary artery bypass surgery

- TIMI flow $<2$ of the infarct-related artery after PCI

- Unstable clinical status (hemodynamic or electrical instability)

- Significant coronary calcification or tortuosity deemed to preclude IVUS, NIRS and OCT evaluation

- Uncontrolled cardiac arrhythmia, defined as recurrent and symptomatic ventricular tachycardia or atrial fibrillation with rapid ventricular response not controlled by medications in the past 3 mo prior to screening

- Severe renal dysfunction, defined by estimated glomerular filtration rate $<30 \mathrm{ml} / \mathrm{min} / 1.73 \mathrm{~m}^{2}$

- Active liver disease or hepatic dysfunction

- Known intolerance to rosuvastatin OR known statin intolerance

- Known allergy to contrast medium, heparin, aspirin, ticagrelor or prasugrel

- Known sensitivity to any substances to be administered, including known statin intolerance

- Patients who previously received alirocumab or other PCSK9 inhibitor

- Patient who received cholesterol ester transfer protein inhibitors in the past 12 mo prior to screening

- Treatment with systemic steroids or systemic cyclosporine in the past 3 mo

- Known active infection or major hematologic, metabolic, or endocrine dysfunction in the judgment of the Investigator

- Planned surgery within 12 mo

- Patients who will not be available for study-required visits in the judgment of the Investigator

- Current enrolment in another investigational device or drug study

- History of cancer within the past 5 y, except for adequately treated basal cell skin cancer, squamous cell skin cancer, or in situ cervical cancer

- Estimated life expectancy less than 1 y

- Female of childbearing potential (age $<50$ y and last menstruation within the last $12 \mathrm{mo}$ ), who did not undergo tubal ligation, ovariectomy or hysterectomy.

NCT03067844) is an investigator-initiated, European, multicentre, double-blind, placebo-controlled, randomized, superiority study which evaluates the effect of alirocumab on coronary atherosclerosis by multimodality intracoronary imaging in patients presenting with AMI undergoing PCI.

Patients who underwent clinically indicated PCI for AMI (ST-elevation or non-ST-elevation myocardial infarction) were screened for clinical and anatomic eligibility for study participation as outlined in Table I. In brief, patients were eligible if they met the following criteria:

(i) Suitability for intracoronary imaging of two noninfarct related arteries (non-IRAs) without signifi- cant obstructive atherosclerotic disease (visual estimate $<50 \%$ angiographic diameter stenosis) but angiographic evidence of atherosclerosis ( $>20 \%$ stenosis).

(ii) LDL-C level $\geq 125 \mathrm{mg} / \mathrm{dL}(3.2 \mathrm{mmol} / \mathrm{L})$ if patients were stain-naïve or had not been on a stable $(\geq 4$ wk) statin regimen at the time of screening; or LCL-C $\geq 70 \mathrm{mg} / \mathrm{dL}(1.8 \mathrm{mmol} / \mathrm{L})$ if patients were on an unchanged statin treatment for $\geq 4$ weeks prior to study enrolment. Among patients considered to be potential study candidates based on all other inclusion and exclusion criteria, LDL-C levels were measured with a rapid test from blood samples (fasting or non-fasting) drawn after the diagnostic 
angiography and prior to PCI. For this purpose, a validated, point-of-care assay (CardioChek PA Silver, Polymer Technology Systems, Indianapolis IN, USA) was available at each participating study centre for prompt measurement of LDL-C levels to determine eligibility for enrolment ${ }^{26}$.

The study is conducted at 9 centres in Switzerland (5), Austria (1), Denmark (1) and the Netherlands (2). A total of 300 patients were randomized in a 1:1 ratio to either alirocumab subcutaneous $150 \mathrm{mg}$ biweekly or matching placebo between May 09, 2017 and October 07, 2020. A CONSORT flow diagram is provided in Supplementary Figure I. Following screening, enrolment and randomization (baseline, day 1), the total study duration for each individual patient will amount to 52 weeks consisting of a 50-week treatment period and a 2 -week period between completion of the treatment period and final follow-up. Study visits will occur at weeks 2, 4, 24 and 52 and telephone follow-up at week 8, 12, 36 and 48. The first administration of the study drug is performed as soon as possible during hospitalization for the index AMI, and the subsequent administrations are done at the week-2 and week- 4 study visits. Afterwards, the study drug will be self-injected by patients at home and documented in a patient logbook. Baseline and follow-up multimodality imaging will be performed at day 1 and week 52, respectively. All patients will receive a protocol-mandated high-intensity statin treatment with rosuvastatin $20 \mathrm{mg} / \mathrm{d}$ without change in dose or type of statin during the entire duration of the study. Patients and treating physicians should refrain from LDL-C measurements throughout the whole study time to maintain blinding. In case of statin intolerance, a standard statin dose reduction algorithm has been implemented. Figure I provides a study design overview and treatment schema of the PACMANAMI trial.

\section{Justification of LDL-C criteria for eligibility}

The rationale for the LDL-C eligibility thresholds in conjunction with pre-enrolment statin treatment status is in line with previous studies using PCSK9 inhibitors, accounting for specific patient selection criteria uniquely applicable in the present study. In previous studies, PCSK9 inhibitors were administered either on top of maximum tolerated statin therapy if LDL-C levels were above target levels as determined by individual patient cardiovascular risk after a lipid-stabilizing period; or instead of a statin (eg, due to statin intolerance). In contrast, in the present study, a lipid-stabilizing phase is not feasible as a result of the enrolment of patients in the acute setting of AMI, and due to the performance of the baseline intracoronary imaging evaluation (a prerequisite to proceed to randomization) during the clinically indicated cardiac catheterization in the acute clinical setting. This study will therefore require that pa- tients have LDL-C levels at screening that are either above the guideline-recommended target of $70 \mathrm{mg} / \mathrm{dL}$ $(1.8 \mathrm{mmol} / \mathrm{L})^{27}$ while on prior stable statin treatment, or are not projected to be lowered below the target for patients not on prior statin treatment. For the latter group, patients with an LDL-C $\geq 125 \mathrm{mg} / \mathrm{dL}(\geq 3.2$ $\mathrm{mmol} / \mathrm{L})$ would remain above target $(>1.8 \mathrm{mmol} / \mathrm{l}) \mathrm{de}-$ spite the protocol-mandated background statin therapy, considering the average $43 \%$ LDL-C reduction reportedly achievable with rosuvastatin $20 \mathrm{mg}^{3}$

\section{Study endpoints}

The PACMAN-AMI randomized trial will evaluate the effect of the PCSK9 inhibitor alirocumab on the change in (Table II):

- IVUS-derived percent atheroma volume (PAV) (primary endpoint)

- NIRS-derived maximum lipid core burden index within $4 \mathrm{~mm}$ (maxLCBI $4 \mathrm{~mm}$ ) (powered secondary endpoint)

- OCT-derived minimal fibrous cap thickness (FCT) (powered secondary endpoint)

Multimodality imaging will be performed at baseline (day 1; time point of cardiac catheterization for the index AMI) and at 52 weeks in the two non-IRAs. Figure II illustrates the assessment of the three imaging endpoints.

\section{Acquisition of IVUS-NIRS and OCT imaging}

Following the completion of coronary angiography and the qualifying PCI procedure on day 1, patients underwent ad hoc intracoronary imaging first with IVUS-NIRS followed by OCT of the two non-IRAs immediately after PCI. The aim was to acquire a major native coronary artery segment between two landmarks that exceeded $50 \mathrm{~mm}$ in length and had angiographic evidence of atherosclerosis but without angiographically significant stenosis (ie, $<50 \%$ ). The regions of interest (ROI) were selected between two sidebranches (ie, a distal sidebranch, and the distal left main or the RCA ostium)

After 52 weeks, patients will undergo intracoronary imaging using IVUS-NIRS and OCT of the same ROI imaged at baseline, as well as imaging of the stented segment by OCT only.

The combined 3.2 French IVUS-NIRS catheter $40 \mathrm{MHz}$ INSIGHT TVC-C195-22 (Intraredx, Bulington, MA, USA) was used for all baseline imaging procedures from study onset until 2019, and any of the serial follow-up procedures at follow-up, and the $50 \mathrm{MHz}$ INSIGHT XB, TVC.C195-32 is used thereafter for all baseline procedures and the serial procedure at follow-up. The IVUSNIRS catheter is advanced beyond the distal landmark and a motorized pullback at a speed of $0.5 \mathrm{~mm} / \mathrm{s}$ and 240 rotations/min is performed after the administration of 100 to $200 \mu \mathrm{g}$ intracoronary nitroglycerine. Serial 


\section{Figure I}

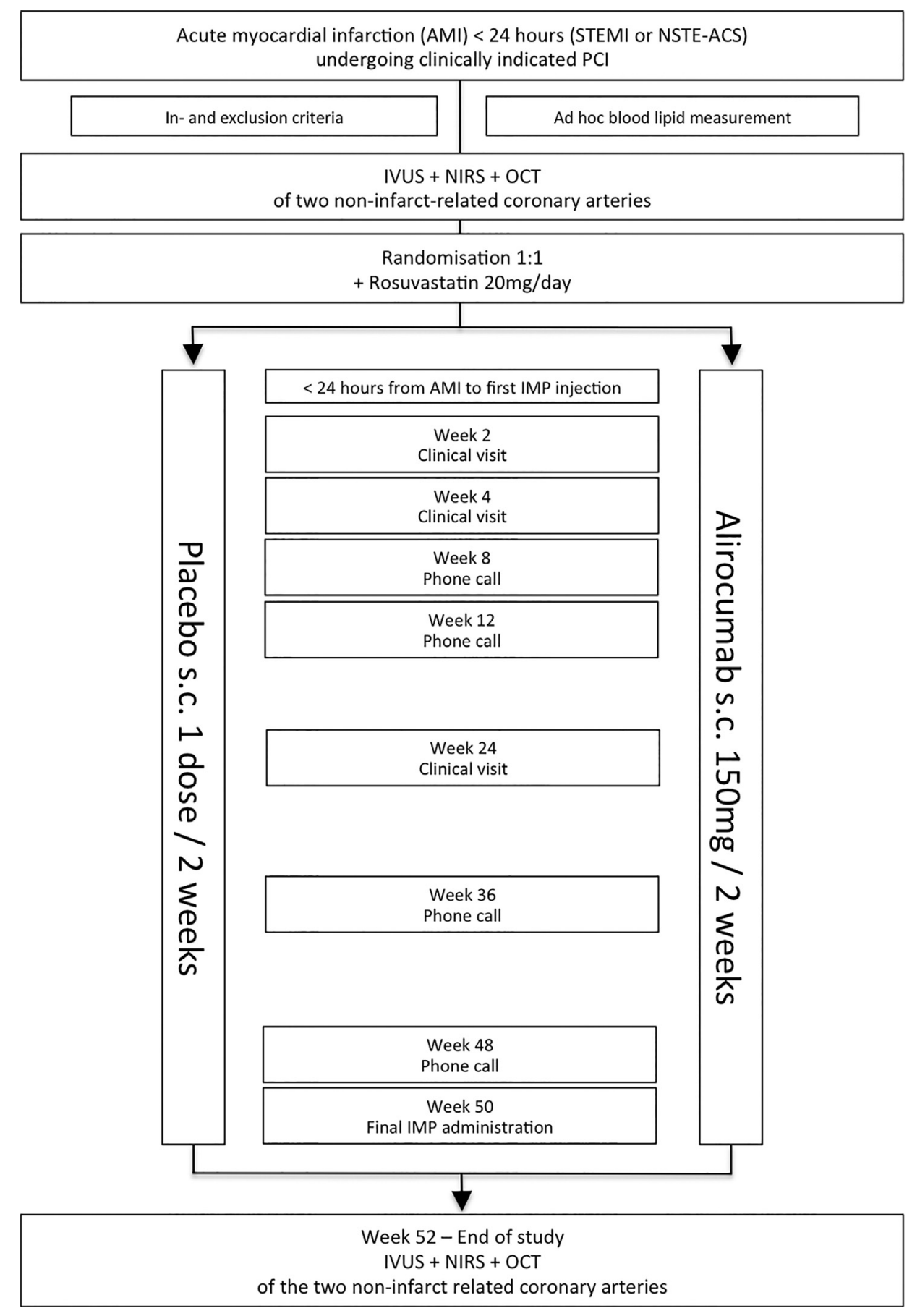

Study design and treatment schema of PACMAN-AMI.

IVUS-NIRS are performed by the same catheter type. Pullbacks are transferred via a dedicated image transfer system (AGmednet) to the independent Core Laboratory (Cardialysis BV, Rotterdam, The Netherlands) for quality control, blinding, and analysis.
OCT imaging will be performed using a frequencydomain OCT system (ILUMIEN OPTIS (St. Jude Medical, St. Paul, MN, USA). After administration of intracoronary nitroglycerin, an automatic pullback OCT imaging is performed using a 2.7 French C7 Dragonfly imaging catheter 


\section{Figure II}
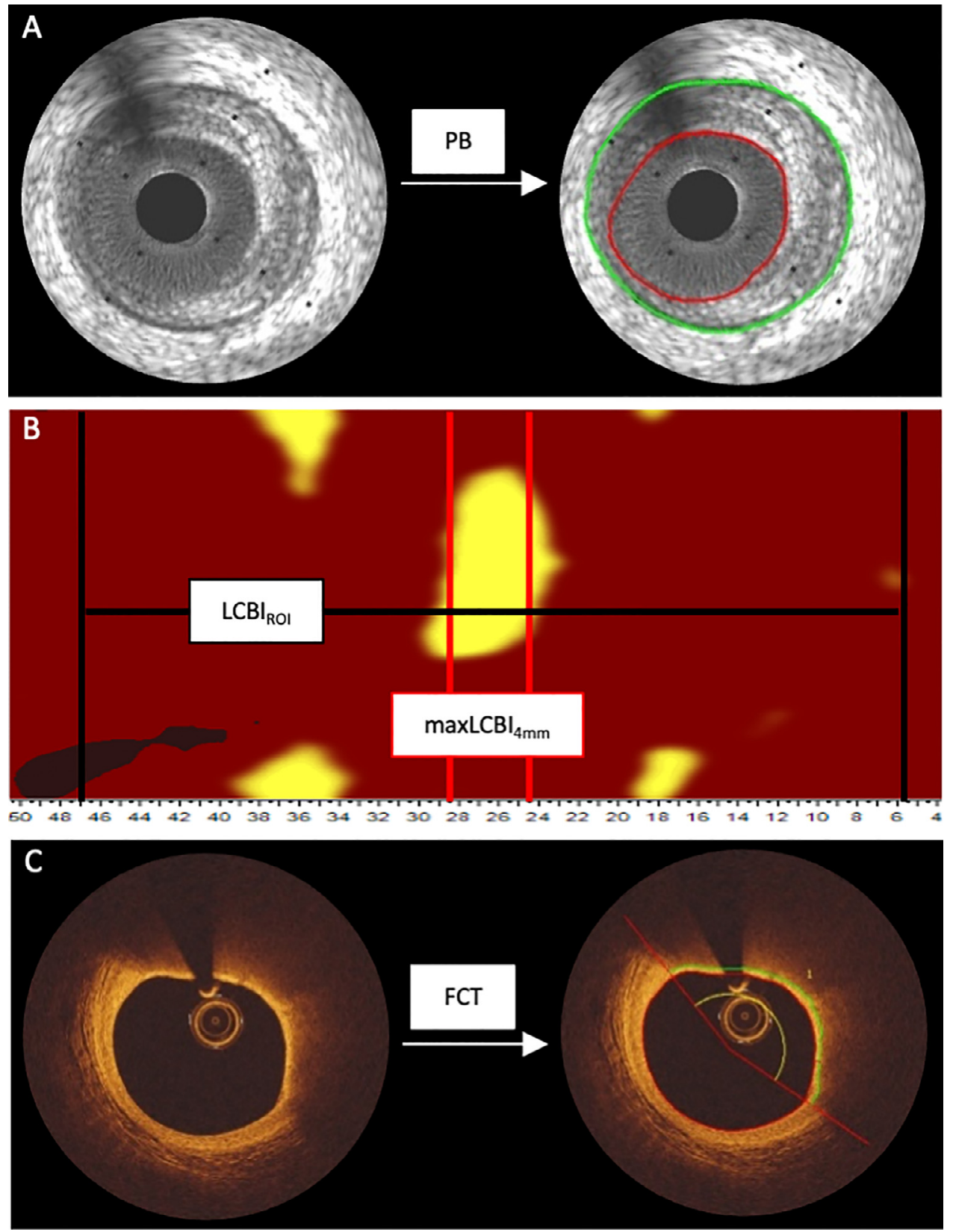

IVUS, NIRS and OCT analyses. (A) Cross sectional IVUS image of a coronary artery. The lumen (red) and external elastic membrane (green) is drawn for plaque burden calculation. (B) Chemogram of a non-infarct related artery. The $\mathrm{LCBI}_{\mathrm{ROI}}$ (indicated by a black line) and maxLCBI $4 \mathrm{~mm}$ (indicated by a red line) of the region of interest is measured. (C) Cross section OCT image of a coronary artery. The lipid angle (red) is drawn and FCT (green line) is measured by a validated semi-automated method. PB, plaque burden, FCT, fibrous cap thickness, LCBI ROI, lipid core burden of the region of interest, $\operatorname{maxLCBI}_{4 \mathrm{~mm}}, 4 \mathrm{~mm}$ segment with the maximum amount of lipid core burden index within the region of interest (color version of figure is available online.) 
Table II. Summary of PACMAN-AMI endpoints

\section{Primary endpoint \\ Powered secondary endpoints}

Secondary endpoints

Secondary clinical endpoints

Safety endpoints
IVUS

- Changes in PAV by greyscale IVUS from baseline to wk 52

NIRS

- Change in $\operatorname{maxLCBI}_{4 \mathrm{~mm}}$ by NIRS from baseline to wk 52

OCT

- Change in minimal fibrous cap thickness by OCT from baseline to wk 52

IVUS

- Change in normalized total atheroma volume (NTAV) by IVUS from baseline to wk 52

NIRS

- Change in $\mathrm{LCBI}_{\mathrm{ROI}}$ by NIRS from baseline to wk 52

OCT

- Change in mean fibrous cap thickness by OCT from baseline to wk 52

- Change in average angular extension of macrophages by OCT from baseline to wk 52

Biomarkers

- Change in lipid levels (cholesterol, LDL-C, HDL-C, Lp(a), triglycerides, non-HDL-C, Apo B, Apo A-1, ratio Apo B/Apo A-1, Apo C-III, Lp(a))

- Inflammatory biomarkers (hs-CRP, TNFa, IL I b, IL-6, MPO, cystatine, SIRT1, SIRT6) and other selected biomarkers (hs-TnT, NT-pro-BNP)

- Any death

- Cardiac death

- Non-fatal myocardial infarction

- Ischemia-driven coronary revascularization (Target lesion revascularization, target vessel revascularization, non-target vessel revascularization)

- Ischemic stroke/TIA

- Adverse events (AEs), serious adverse events (SAEs), adverse events of special interest (AESIs)
(Dragon Fly Duo, LightLab, St. Jude Medical, St. Paul, MN, USA). Pullbacks are recorded during automated contrast injection with and automated injector such as ACIST for quality reason with an injection rate of $>5.0 \mathrm{ml} / \mathrm{s}$ for the left coronary artery and $>4.0 \mathrm{ml} / \mathrm{s}$ for the right coronary artery depending on the vessel size. OCT pullbacks will be sent via AGmednet to the OCT Corelab at the University Hospital of Bern for quality control, blinding and analysis.

\section{Analyses of IVUS-NIRS and OCT imaging}

- IVUS: The independent Corelab Cardialysis, Rotterdam, NL, will randomly allocate a code to the baseline and follow-up pullbacks in order to ensure blinding of the analysts to the temporal sequence and treatment allocation of paired images. The largest common ROI available from the two serial recordings will be assessed with the help of dedicated matching software and identified as much common matching points within the pullbacks (eg, side branches, calcifications). Within the matched
ROI, the lumen and external elastic membrane will be measured every $1 \mathrm{~mm}$ (QIVUS Research Edition 3.1.12.0, Medis, Leiden, The Netherlands). IVUS analyses will be performed as previously described. ${ }^{28}$ The arterial lumen and external elastic membrane borders will be segmented from digitized IVUS images. The primary IVUS-derived parameter will be PAV (=plaque burden) according to the following equation:

$$
P A V=\frac{\Sigma(\text { EEMCSA }- \text { LumenCSA })}{\Sigma \text { EEMCSA }} \times 100
$$

where external elastic membrane ${ }_{\mathrm{CSA}}$ is the external elastic membrane cross-sectional area and Lumen $_{\mathrm{CSA}}$ is the luminal cross-sectional area. The primary endpoint will be the change in PAV between baseline and follow-up. Normalized total atheroma volume (normalized TAV) will be assessed as secondary endpoint.

- NIRS: Spectroscopic information will be obtained from the IVUS ROI and displayed as NIRS "chemogram". The presence of lipid core burden 
will be assessed and quantified by the lipid core burden index (LCBI), a quantitative summary metric of lipid core presence in a given longitudinal region. LCBI is computed as the fraction of valid pixels within the study region that exceed a lipidcore plaque (LCP) probability of 0.6 , multiplied by $1000 .{ }^{29}$ Thus, LCBI is measured on a scale from 0 to 1000. For each ROI, the $4 \mathrm{~mm}$ segment with maximum LCBI ( $\left.\operatorname{maxLCBI}_{4 \mathrm{~mm}}\right)$ and the LCBI over the total length $\left(\mathrm{LCBI}_{\mathrm{ROI}}\right)$ will be measured. ${ }^{11,12,29}$ Analyses will be performed offline using QIVUS Research Edition 3.1.12.0 software at the independent Corelab Cardialysis, Rotterdam, NL.

The powered secondary endpoint will be the change in maxLCBI $_{4} \mathrm{~mm}$ between baseline and follow-up investigation within the same ROI. LCBI $_{\text {ROI }}$ will be assessed as the secondary endpoint.

- OCT: OCT images will be analyzed offline at every single frame $(0.4 \mathrm{~mm})$ within matched ROI using proprietary software (QCU-CMS version 4.69 software, Medis, Leiden, The Netherlands) blinded to time-point of assessment as previously reported by our group. ${ }^{30-32}$ Each frame will be classified based on a hierarchical approach as normal vessel, fibrous plaque, fibrocalcific plaque, and fibroatheroma. A fibroatheroma is defined as a plaque with evidence of lipid-pool $>90^{\circ}$ which is characterized by a poorly defined or diffuse border between the signalpoor region and surrounding tissue without lateral delineation. ${ }^{21}$ For every frame classified as fibroatheroma, we will measure the FCT, defined as the signal-rich tissue layer overlying the lipid pool by using a previously validated, highly reproducible, semi-automated method. ${ }^{33}$ In addition, the lipid arc and lipid length (defined as the length of plaque with $>90^{\circ}$ of lipid measured on the longitudinal view) will be assessed.

The powered secondary endpoint for the OCT analysis is the change in minimal FCT from baseline to week 52. Change in mean FCT and average angular extension of macrophages will be assessed as the secondary endpoints. Additional endpoints are change in mean FCT, lipid pool arc and plaque type by OCT.

\section{PACMAN-AMI substudies}

Several substudies will investigate the impact of alirocumab on endothelial and platelet function, shear stress, lipidomics, neutrophil extracellular traps, neoatherosclerosis in the stent implanted in the culprit lesion, changes in quantitative flow ratio within the ROI, and statin adherence. Table III provides an overview of the PACMAN-AMI substudies timeline with the individual time point of assessment. An overview on the aims and endpoints of the PACMAN-AMI related substudies is shown in Supplementary Table I.
Sample size determination and statistical analysis: Power analysis for change in PAV (primary endpoint)

PACMAN AMI is a superiority trial powered for the primary and two secondary endpoints. In the final power calculation, incorporating also the results of the GLAGOV trial $^{23}$, we assume a placebo-controlled PAV change $-1.3 \%$ at 52 weeks; standard deviation of $3.4 \%$ (as consensus from SATURN ${ }^{34}: 3.0 \%$, IBIS $4^{28}: 3.4 \%$ ASTEROID $^{16}: 4.0 \%$ ); and intraclass correlation coefficient (ICC) of approximately 0.435 (estimated from IBIS 4 data). ${ }^{28}$ We expect $\mathrm{m}=2.0$ vessels per patients to be analyzed. The design effect is calculated by $\mathrm{D}=1+\mathrm{ICC}(\mathrm{m}-1)$. If dropout was ignored, a total sample size of 264 patients would be required to reach a statistical power of $80 \%$ at a significance level of $\alpha=5 \%$ using a two-sided test. Anticipating a dropout rate of $10 \%$ at the 52-week imaging follow-up, a total of $n=294$ patients should be recruited (147/arm).

Power analysis for change in max $\mathrm{LCBI}_{4} \mathrm{~mm}$ (powered secondary endpoint)

For the change in $\operatorname{maxLCBI}_{4} \mathrm{~mm}$ between baseline and 52 weeks we assume: (i) difference between the two treatment arms of 193.3 based on the observed difference in the YELLOW I trial ${ }^{20}$ and the expected reduction in LDL-C in PACMAN-AMI $[-40 \%$ in the placebo (rosuvastatin-only) and $-75 \%$ in the active (alirocumab plus rosuvastaton) group], (ii) standard deviation of 220 (estimated from the Lipid-Rich Plaque study ${ }^{14}$ ) and iii) dropout rate of $10 \%$ at the 52-week imaging followup. Considering a total number of enrolled patients of $n=294$, a significance level of alpha $=2.5 \%$ using a two-sided test, PACMAN would provide a power of $>95 \%$ to detect the expected difference in the change in $\operatorname{maxLCBI}_{4} \mathrm{~mm}$ of 193.3 between placebo and alirocumab if it was tested independently.

\section{Power analysis for change in minimal FCT (powered secondary endpoint)}

For the change in minimal FCT between baseline and 52 weeks we assume: (i) difference between the alirocumab and placebo arm of $19.8 \mu \mathrm{m}$ for the change in minimal FCT based on the observed difference in IBIS$4^{21}$ and the expected reduction in LDL-C in PACMANAMI (-40\% in Placebo and $-75 \%$ in PCSK9 group), (ii) standard deviation of 44.8 (calculated from IBIS-4); (iii) intracluster correlation coefficient of 0.57 (estimated from IBIS-4); (iv) $\mathrm{m}=1.59$ vessels per patient (ie, with fibroatheroma according to the PACMAN-AMI Matching Substudy ${ }^{35}$ ), (v) and a rate of $72 \%$ having any fibroatheroma by OCT (PACMAN-AMI Matching Substudy). Considering a dropout rate of $10 \%$ at the 52 -week imaging follow-up, a total number of enrolled patients of $\mathrm{n}=294$, a significance level of alpha $=2.5 \%$ using a twosided test, the study would provide $85 \%$ power to detect the expected difference in the change in minimal FCT of 
Table III. PACMAN-AMI substudies timeline

\begin{tabular}{|c|c|c|c|c|c|c|c|c|c|}
\hline \multirow[b]{3}{*}{ Substudy } & \multicolumn{9}{|l|}{ Time Point } \\
\hline & \multicolumn{4}{|c|}{ Day 1: In-hospital } & \multirow[b]{2}{*}{$\begin{array}{l}\text { Wk } 4 \\
\text { clinical } \\
\text { visit }\end{array}$} & \multirow{2}{*}{$\begin{array}{l}\text { End of study } \\
\text { Wk } 52 \text { clinical } \\
\text { visit } \\
\text { IVUS/NIRS + OCT }\end{array}$} & \multicolumn{3}{|c|}{ Extended follow-up } \\
\hline & Before $\mathrm{PCl}$ & $\begin{array}{l}\text { Baseline } \\
\text { IVUS/NIRS } \\
+\mathrm{OCT}\end{array}$ & $\begin{array}{l}\text { Before first } \\
\text { IMP } \\
\text { injection }\end{array}$ & $\begin{array}{l}12-24 \text { hours } \\
\text { after first IMP } \\
\text { injection }\end{array}$ & & & $\begin{array}{l}2 y \\
\text { phone } \\
\text { call }\end{array}$ & $\begin{array}{l}5 y \\
\text { phone } \\
\text { call }\end{array}$ & $\begin{array}{l}10 y \\
\text { phone } \\
\text { call }\end{array}$ \\
\hline (A) Biobank & $x$ & & & & $x$ & $x$ & & & \\
\hline (B) Therapeutic drug monitoring & $x$ & & & & $x$ & $x$ & & & \\
\hline (C) Platelet function & & & $x$ & $x$ & $x$ & $x$ & & & \\
\hline (D) Endothelial function & & & & & $x$ & $\times$ & & & \\
\hline (E) Lipidomics & & & $x$ & & & $x$ & & & \\
\hline (F) Matching & & $x$ & & & & & & & \\
\hline (G) Endothelial shear stress & & $x$ & & & & $x$ & & & \\
\hline (H) Neoatherosclerosis & & & & & & $x$ & & & \\
\hline (I) Neutrophil extracellular trap & $x$ & & $x$ & & $x$ & $x$ & & & \\
\hline (J) 3D carotid ultrasound & & & & $x$ & & $x$ & & & \\
\hline (K) PET/CT & & & & $x$ & & $x$ & & & \\
\hline (L) Quantitative flow ratio & & $x$ & & & & $x$ & & & \\
\hline (M) Legacy effect & & & & & & & $x$ & $x$ & $x$ \\
\hline
\end{tabular}

Footnote: Aim of each substudy; (A) Biobank: To assess the effects of alirocumab on the change in lipid biomarkers, inflammatory biomarkers, and to explore possible associations of biomarkers with changes in coronary plaque characteristics and to improve risk stratification for PCSK9-targeted lipid-lowering therapy, (B) Therapeutic drug monitoring: To assess the adherence, (C) Platelet function: To assess the effect of alirocumab on platelet function as assessed by VerifyNow, (D) Endothelial function: To assess the effect of alirocumab on endothelial function by using flow-mediated dilation (FMD) measurement, (E) Lipidomics: To assess the impact of alirocumab in apolipoproteins by using mass spectrometry and to explore a correlation between changes in apolipoprotein levels with changes in coronary plaque characteristics, (F) Matching: To assess the morphological features of NIRS-defined lipid-rich plaques by using OCT and IVUS, (G) Endothelial shear stress: To assess the impact of alirocumab on endothelial shear stress distribution and plaque structural stress properties, $(H)$ Neoatherosclerosis: To assess the effect of alirocumab on neoatherosclerosis formation in the neointima of the culprit stent, (I) Neutrophilic extracellular traps - DNase activity: To assess the impact of alirocumab on neutrophil activation and receptor expression, the formation of neutrophil extraellular traps, and DNase activity, (J) 3D carotic ultrasound: To evaluate the effect of PCSK9 inhibitor Alirocumab as compared with placebo on the change in PAV and echogenicity in carotid arteries, (K) PET/CT: To investigate the effect of alirocumab on vascular inflammation as assessed by positron emission tomography computer tomography (PET/CT) of the aorta, (L) Quantitative flow ratio: To assess the effect of alirocumab on the change in quantitative flow ratio (QFR) and maximum diameter stenosis (\%DS) in non-IRA, (M) Legacy effect: To assess the clinical effects of alirocumab after 2, 5, and 10 y after enrolment.

$19.8 \mu \mathrm{m}$ between placebo and alirocumab if it was tested independently.

\section{Statistical analyses of the primary and powered secondary endpoints}

The primary endpoint (percentage change in PAV) and the two powered secondary endpoints (change in $\operatorname{maxLCBI}_{4} \mathrm{~mm}$ and change in minimal FCT) will be tested independently. The resulting $\mathrm{p}$-values will be interpreted using a gatekeeping procedure whereby the primary endpoint will first be tested at an alpha level $=0.05$. If a $P$-value $\geq 0.05$ is found for the primary endpoint, then the $P$-values for the powered secondary endpoints will not be interpreted. If a $P$-value $<0.05$ is found for the primary endpoint, alpha will be equally split across the two powered secondary endpoints $($ alpha $=0.025)$ using Bonferroni correction; ie, for the secondary endpoints significance will be achieved at the alpha $=0.025$ level

\section{Discussion}

The PACMAN-AMI trial uniquely investigates the effect of PCSK9 inhibition on plaque morphology and composition, as defined by serial multimodality intracoronary imaging, in the two non-IRAs of patients with AMI including IVUS-derived plaque burden, NIRS-derived lipid core burden index, and OCT-derived fibrous cap thickness. These morphological and compositional plaque characteristics have been consistently related to plaque vulnerability and rupture in pathological studies and with future cardiac events in prospective trials. ${ }^{8,14}$ The PACMANAMI trial aims to provide further mechanistic insights on the effects of highly potent LDL-C reduction on coronary atherosclerosis including plaque volume, composition and microstructural plaque characteristics against a background of evidence demonstrating the efficacy of PCSK9 inhibitors in reducing LDL-C levels and improving clinical outcomes. ${ }^{5,6}$

Landmark IVUS studies ${ }^{16,17}$ have shown a favorable effect of statins on plaque burden. Moreover, statins have been shown to increase the OCT-derived fibrous cap thickness ${ }^{21}$ and decrease the NIRS-derived lipid content. ${ }^{20}$ The Identification of PCSK9 expression in human atherosclerotic plaques has raised the translational hypothesis of a direct favorable effect of PCSK9 inhibition on plaque biology beyond the potent LDL-C-lowering effect. $^{36}$ The GLAGOV trial ${ }^{23}$ evaluated PCSK9 inhibition on atheroma volume as assessed by IVUS in statintreated, stable CAD patients, which resulted in reduction of plaque burden by $-1.0 \%(-1.3$ to $-0.6 \%, P<0.01)$ as compared to placebo. However, there was no change 
in plaque composition as assessed by virtual histology, likely indicating limited value of virtual histology-IVUS for the evaluation of plaque morphology. ${ }^{24}$

In comparison with previous evidence of intravascular imaging studies assessing the effect of lipid-lowering therapy on atherosclerosis, the PACMAN-AMI trial will provide further insights along the following lines:

- While previous studies (of statins and evolocumab) were limited to one intravascular imaging modality, the PACMAN-AMI trial uses a multimodality imaging approach of intracoronary imaging techniques with IVUS, NIRS and OCT. Thereby, the PACMANAMI trial provides incremental insights on the efficacy of a PCSK9 antibody on various aspects of atherosclerosis including plaque volume, composition, and microstructure.

- The GLAGOV trial ${ }^{23}$ mainly enrolled patients with stable or stabilized CAD, while the PACMAN-AMI trial enrolls patients with AMI, a high-risk vulnerable patient population. While all patients enrolled in the GLAGOV trial were on a statin treatment, most patients in the PACMAN trial are statin-naive. The advantage of a non-lipid-stabilizing phase will provide the possibility to uniquely investigate changes in coronary atheroma burden, composition and microstructure in response to newly initiated statin treatment alone vs. a treatment combined with a PCSK9 inhibitor in high-risk patients with AMI. Acute myocardial infarction causes a systemic inflammatory reaction and an increase of PCSK9 levels, which promotes inflammation and thereby promotes coronary plaque vulnerability through pro-inflammatory pathways, direct modification of plaque composition and activation of prothrombotic mechanisms as demonstrated in preclinical models. ${ }^{37}$ Hence, we hypothesize that early $(<24$ $h$ following index PCI) application of PCSK9 antibodies during the acute setting of a myocardial infarction may lead to an early plaque stabilization effect via anti-inflammatory and antithrombotic mechanisms. In addition, patients with AMI are known to harbour more vulnerable plaques in their non-infarct related arteries compared to stable CAD patients ${ }^{38,39}$, thereby indicating an ideal population to study the PCSK9-inhibitor-mediated effects on atherosclerosis. As an additional feature, a multi-vessel assessment will be obtained in the proposed study, as plaque progression in the entire coronary artery tree is of greater interest compared to assessment of only a selected single arterial segment. ${ }^{40}$ Cardiovascular events are not limited to one single vessel segment and in this study we will focus specifically on selecting the clinically most relevant vessel regions, that is, according to previous pathology studies ${ }^{41,42}$, the proximal portions of the major coronary arteries where most rupture-prone thin-cap fibroatheromas are located and where the consequences of a myocardial infarction are most pronounced based on the large amount of myocardium at risk.

- Several substudies conducted within the scope of the PACMAN-AMI trial will provide valuable insights into effects of alirocumab on (I) lipid biomarkers, inflammatory biomarkers and associations of biomarkers with changes in coronary plaque characteristics,; (II) platelet function; (III) endothelial function; (IV) shear stress; (V) neoatherosclerosis formation; (VI) neutrophil activation and the formation of neutrophil extracellular traps; (VII) PAV and echogenicity in carotid arteries; (VIII) PET/CTderived atherosclerosis; and (IX) quantitative flow ratio of angiographically non-significant stenosis. These studies will further enrich our knowledge of potential pleiotropic effects of PCSK9 inhibition.

\section{Summary}

The PACMAN-AMI trial is an investigator-initiated, multicenter, double-blind, placebo-controlled, randomized superiority study enrolling a total of 300 patients to determine the effect of alirocumab on IVUS-derived PAV, NIRS-derived maxLCBI $4 \mathrm{~mm}$ and OCT-derived minimal FCT in the two non-IRAs in patients presenting with AMI. Enrolment was completed in October 2020, and study completion is expected in Q4 2021.

\section{Funding}

The study is supported by a grant to Bern University Hospital provided by Regeneron, Sanofi, and Infraredx. The grant providers were not involved in protocol writing, data acquisition, storage and analysis. Regeneron provided alirocumab and the matching placebo free of charge. One employee of Sanofi (DS) contributed to the trial conception, and also provided expertise on the investigational medicinal product, study material, and drug-related assay and equipment.

\section{Disclosures}

KCK received research grants to the institution by Amgen and consultation or speaker fees by Amgen and Sanofi. SB reports a research grant to the institution from Medis Medical Imaging Systems. JD received institutional grant/research support from Astra Zeneca, Abbott Vascular, Boston Scientific, ACIST Medical, Medtronic, Pie Medical, and ReCor medical, and consultancy and speaker fees from Abiomed, ACIST medical, Boston Scientific, PulseCath, ReCor Medical, Pie Medical and Medtronic. CMM received research grants to the institution by Sanofi, Swiss Heart Foundation and Swiss National Science Foundation and consultation or speaker 
fees by Novartis, Daiichi Sankyo and MSD. CMM is a paid member of the section careers of the Swiss National Science Foundation. DS is an employee of Sanofi. TE has received speakers/advisory board fee from Abbott Vascular, Boston Scientific, Bayer, Novo Nordisk. SB reports a research grant to the institution from Medis Medical Imaging Systems. SW reports research and educational grants to the institution from Abbott, Amgen, BMS, Bayer, Boston Scientific, Biotronik, Cardinal Health, CardioValve, CSL Behring, Daiichi Sankyo, Edwards Lifesciences, Johnson\&Johnson, Medtronic, Querbet, Polares, Sanofi, Terumo, Sinomed. SW serves as unpaid member of the steering/executive group of trials funded by Abbott, Abiomed, Amgen, BMS, Boston Scientific, Biotronik, Cardiovalve, Edwards Lifesciences, MedAlliance, Medtronic, Novartis, Polares, Sinomed, V-Wave and Xeltis, but has not received personal payments by pharmaceutical companies or device manufacturers. He is also member of the steering/executive committee group of several investigated-initiated trials that receive funding by industry without impact on his personal remuneration. SW is an unpaid member of the Pfizer Research Award selection committee in Switzerland. LR has received research grants to the institution by Abbott Vascular, Biotronik, BostonScientific, Heartflow, Sanofi, and Regeneron and consultation or speaker fees by Abbott vascular, Abbott structural, AstraZeneca, Amgen, Canon, Infraredx, Sanofi, Vifor. RvG received research grants to the institution by AstraZeneca, Amgen and InfraRedx. All other authors don't report any conflict of interest.

\section{Supplementary materials}

Supplementary material associated with this article can be found, in the online version, at doi:10.1016/j.ahj. 2021.04.006.

\section{References}

1. Baigent C, Blackwell L, Emberson J, et al. Efficacy and safety of more intensive lowering of LDL cholesterol: a meta-analysis of data from 170000 participants in 26 randomised trials. The Lancet 2010;376:1670-81

2. Koskinas KC, Siontis GCM, Piccolo R, et al. Effect of statins and non-statin LDL-lowering medications on cardiovascular outcomes in secondary prevention: A meta-analysis of randomized trials. Eur Heart J 2018;39:1 172-80

3. Mach F, Baigent C, Catapano AL, et al. 2019 ESC/EAS Guidelines for the management of dyslipidaemias: lipid modification to reduce cardiovascular risk. Eur Heart J 2020;41:111-88.

4. Koskinas KC, Gencer B, Nanchen D, et al. Eligibility for PCSK9 inhibitors based on the 2019 ESC/EAS and 2018 ACC/AHA guidelines. Eur J Prev Cardiol $2021 ; 28(1): 59-65$.

5. Sabatine MS, Giugliano RP, Keech AC, et al. Evolocumab and clinical outcomes in patients with cardiovascular disease. New Engl J Med 2017;376:1713-22.
6. Schwartz GG, Steg PG, Szarek M, et al. Alirocumab and cardiovascular outcomes after acute coronary syndrome. New Engl J Med 2018:2097-107.

7. Koskinas KC, Windecker S, Pedrazzini G, et al. Evolocumab for early reduction of LDL cholesterol levels in patients with acute coronary syndromes (EVOPACS). J Am Coll Cardiol 2019;74:2452-62.

8. Koskinas KC, Ughi GJ, Windecker S, et al. Intracoronary imaging of coronary atherosclerosis: validation for diagnosis, prognosis and treatment. European Heart Journal 2016;37:524-35 a-c.

9. Garcia-Garcia HM, Costa MA, Serruys PW. Imaging of coronary atherosclerosis: intravascular ultrasound. Eur Heart J 2010;31:2456-69.

10. Tearney GJ, Regar E, Akasaka T, et al. Consensus standards for acquisition, measurement, and reporting of intravascular optical coherence tomography studies: a report from the International Working Group for Intravascular Optical Coherence Tomography Standardization and Validation. J Am Coll Cardiol 2012;59:1058-72

11. Gardner CM, Tan H, Hull EL, et al. Detection of lipid core coronary plaques in autopsy specimens with a novel catheter-based near-infrared spectroscopy system. JACC Cardiovasc Imaging 2008; 1:638-48.

12. Waxman S, Dixon SR, L'Allier $P$, et al. In vivo validation of a catheter-based near-infrared spectroscopy system for detection of lipid core coronary plaques: initial results of the SPECTACL study. JACC Cardiovasc Imaging 2009;2:858-68.

13. Prati F, Romagnoli E, Gatto L, et al. Relationship between coronary plaque morphology of the left anterior descending artery and 12 months clinical outcome: the CLIMA study. Eur Heart J 2020; 14:383-91

14. Waksman R, Di Mario C, Torguson R, et al. Identification of patients and plaques vulnerable to future coronary events with near-infrared spectroscopy intravascular ultrasound imaging: a prospective, cohort study. The Lancet 2019;394:1629-37.

15. Erlinge $D$, Maehara $A$, Ben-Yehuda $O$, et al. Identification of vulnerable plaques and patients by intracoronary near-infrared spectroscopy and ultrasound (PROSPECT II): a prospective natural history study. The Lancet 2021;397:985-95.

16. Nissen SE, Nicholls SJ, Sipahi I, et al. Effect of very high-intensity statin therapy on regression of coronary atherosclerosis: the ASTEROID trial. JAMA 2006;295:1556-65

17. Nicholls SJ, Ballantyne CM, Barter PJ, et al. Effect of two intensive statin regimens on progression of coronary disease. New Engl J Med 2011;365:2078-87.

18. Crisby M, Nordin-Fredriksson G, Shah PK, et al. Pravastatin treatment increases collagen content and decreases lipid content, inflammation, metalloproteinases, and cell death in human carotid plaques: implications for plaque stabilization. Circulation $2001 ; 103: 926-33$

19. Moreno PR, Kini A. Resolution of inflammation, statins, and plaque regression. JACC Cardiovasc Imaging 2012;5:178-81.

20. Kini AS, Baber U, Kovacic JC, Limaye A, Ali ZA, Sweeny J, et al. Changes in plaque lipid content after short-term intensive versus standard statin therapy: the YELLOW trial (reduction in yellow plaque by aggressive lipid-lowering therapy). J Am Coll Cardiol 2013;62:21-9.

21. Räber L, Koskinas KC, Yamaji K, et al. Changes in coronary plaque composition in patients with acute myocardial infarction treated with high-intensity statin therapy (IBIS-4). JACC: Cardiovasc Imaging 2019;12:1518-28. 
22. Komukai K, Kubo T, Kitabata H, et al. Effect of atorvastatin therapy on fibrous cap thickness in coronary atherosclerotic plaque as assessed by optical coherence tomography: the EASY-FIT study. J Am Coll Cardiol 2014;64:2207-17.

23. Nicholls SJ, Puri R, Anderson T, et al. Effect of evolocumab on progression of coronary disease in statin-treated patients: the GLAGOV randomized clinical trial. JAMA 2016;316 2373.

24. Nicholls SJ, Puri R, Anderson T, et al. Effect of evolocumab on coronary plaque composition. J Am Coll Cardiol 2018;72:2012-2123.

25. Ako J, Hibi K, Tsujita K, et al. Effect of alirocumab on coronary atheroma volume in japanese patients with acute coronary syndrome-the ODYSSEY J-IVUS trial. Circ J 2019;83:2025-33 25.

26. Panz VR, Raal FJ, Paiker J, et al. Performance of the CardioChek PA and Cholestech LDX point-of-care analysers compared to clinical diagnostic laboratory methods for the measurement of lipids. Cardiovasc J S Afr 2005; 16:1 12-17.

27. Piepoli MF, Hoes AW, Agewall S, et al. 2016 European Guidelines on cardiovascular disease prevention in clinical practice: The Sixth Joint Task Force of the European Society of Cardiology and Other Societies on Cardiovascular Disease Prevention in Clinical Practice (constituted by representatives of 10 societies and by invited experts)Developed with the special contribution of the European Association for Cardiovascular Prevention \& Rehabilitation (EACPR). Eur Heart J 2016;37:2315-81.

28. Räber L, Taniwaki M, Zaugg S, et al. Effect of high-intensity statin therapy on atherosclerosis in non-infarct-related coronary arteries (IBIS-4): a serial intravascular ultrasonography study. Eur Heart J 2015;36:490-500.

29. Madder RD, Goldstein JA, Madden SP, et al. Detection by near-infrared spectroscopy of large lipid core plaques at culprit sites in patients with acute ST-segment elevation myocardial infarction. JACC Cardiovasc Interv 2013;6:838-46.

30. Räber L, Zanchin T, Baumgartner S, et al. Differential healing response attributed to culprit lesions of patients with acute coronary syndromes and stable coronary artery after implantation of drug-eluting stents: an optical coherence tomography study. In J Cardiol 2014;173:259-67.

31. Räber L, Heo JH, Radu MD, et al. Offline fusion of co-registered intravascular ultrasound and frequency domain optical coherence tomography images for the analysis of human atherosclerotic plaques. Eurolntervention 2012;8:98-108.
32. Räber L, Baumgartner S, Garcia-Garcia HM, et al. Long-term vascular healing in response to sirolimus- and paclitaxel-eluting stents: an optical coherence tomography study. JACC Cardiovasc Interv 2012;5:946-57.

33. Radu MD, Yamaji K, García-García HM, et al. Variability in the measurement of minimum fibrous cap thickness and reproducibility of fibroatheroma classification by optical coherence tomography using manual versus semi-automatic assessment. Eurolntervention 2016;12:e987-97.

34. Nicholls SJ, Ballantyne CM, Barter PJ, et al. Effect of two intensive statin regimens on progression of coronary disease. New Engl J Med 2011 ; 365:2078-87.

35. Zanchin C, Ueki Y, Losdat S, et al. In vivo relationship between near-infrared spectroscopy-detected lipid-rich plaques and morphological plaque characteristics by optical coherence tomography and intravascular ultrasound: a multimodality intravascular imaging study. Euro Heart J Cardiovasc Imaging 2020.

36. Ferri N, Tibolla G, Pirillo A, et al. Proprotein convertase subtilisin kexin type 9 (PCSK9) secreted by cultured smooth muscle cells reduces macrophages LDLR levels. Atherosclerosis 2012;220:381-6. doi:10.1093/ehjci/jez318.

37. Navarese EP, Kołodziejczak M, Kereiakes DJ, et al. Proprotein convertase subtilisin/kexin type 9 monoclonal antibodies for acute coronary syndrome a narrative review. Ann Intern Med 2016;164:600-7.

38. Hong M-K, Mintz GS, Lee CW, et al. Comparison of coronary plaque rupture between stable angina and acute myocardial infarction: a three-vessel intravascular ultrasound study in 235 patients. Circulation 2004; 1 10:928-33.

39. Puri R, Nissen SE, Shao M, et al. Antiatherosclerotic effects of long-term maximally intensive statin therapy after acute coronary syndrome: insights from study of coronary atheroma by intravascular ultrasound: effect of rosuvastatin versus torvastatin. Arterioscler Thromb Vasc Biol 2014;34:2465-72.

40. Stone GW, Maehara A, Lansky AJ, et al. A prospective natural-history study of coronary atherosclerosis. New Engl J Med $2011 ; 364: 226-35$.

41. Virmani R, Virmani R, Kolodgie FD, et al. Lessons from sudden coronary death. Arterioscler Thromb 2000: 1262-75.

42. Cheruvu PK, Finn AV, Gardner C, et al. Frequency and Distribution of thin-cap fibroatheroma and ruptured plaques in human coronary arteries. a pathologic study. J Am Coll Cardiol 2007;50:940-9. 